\title{
A rapid, high-throughput method for determining chronological lifespan in budding yeast
}

Zachery R. Belak, Troy Harkness, Christopher H. Eskiw

\section{FIGURE S1}

$\neg-2 \%$ GLU CC $\neg-0.2 \%$ GLU CC $\rightarrow-$ Water CC $\neg 2 \%$ GLU RCL $\rightarrow 0.2 \%$ GLU RCL $\neg$ Water RCL

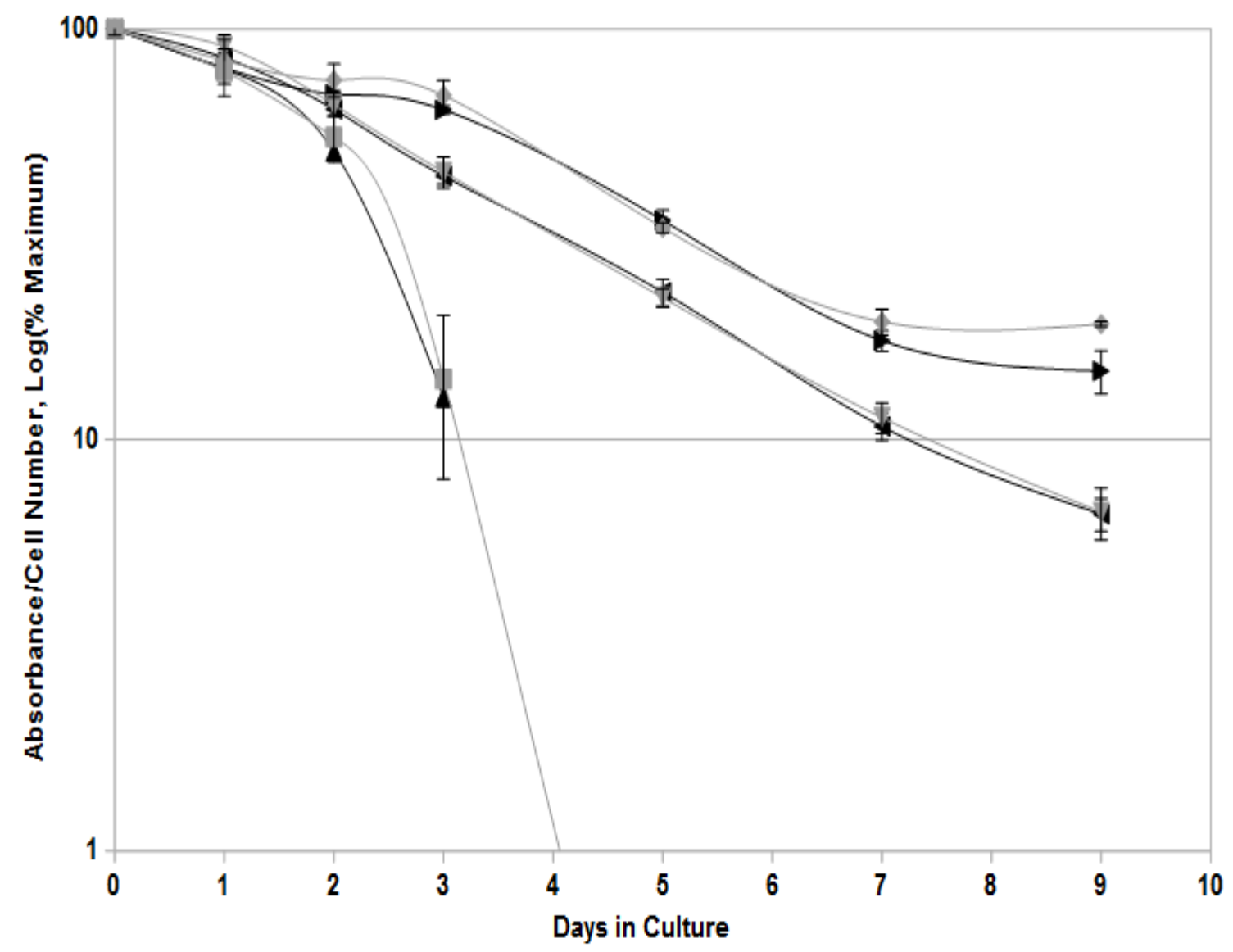

Figure S1. Analysis of yeast chronological lifespan under conditions of caloric restriction, log scale. Stationary phase aging yeast cultures in $\mathrm{CM}$ media containing either $2 \%$ or $0.2 \%$ glucose (GLU), or of cells suspended in water, were incubated for the indicated number of days ( $x$-axis). Each day, an aliquot was removed from the culture and subjected to either the traditional CC assay (grey) or the novel RCL assay described here (black). For CC assays, viability is expressed as logarithm of percent of 
the mean cell number at day 0 , for RCL assays as logarithm of percent of the mean absorbance at day 0 ( $y$-axis). Error bars represent the standard error of three independent replicates.

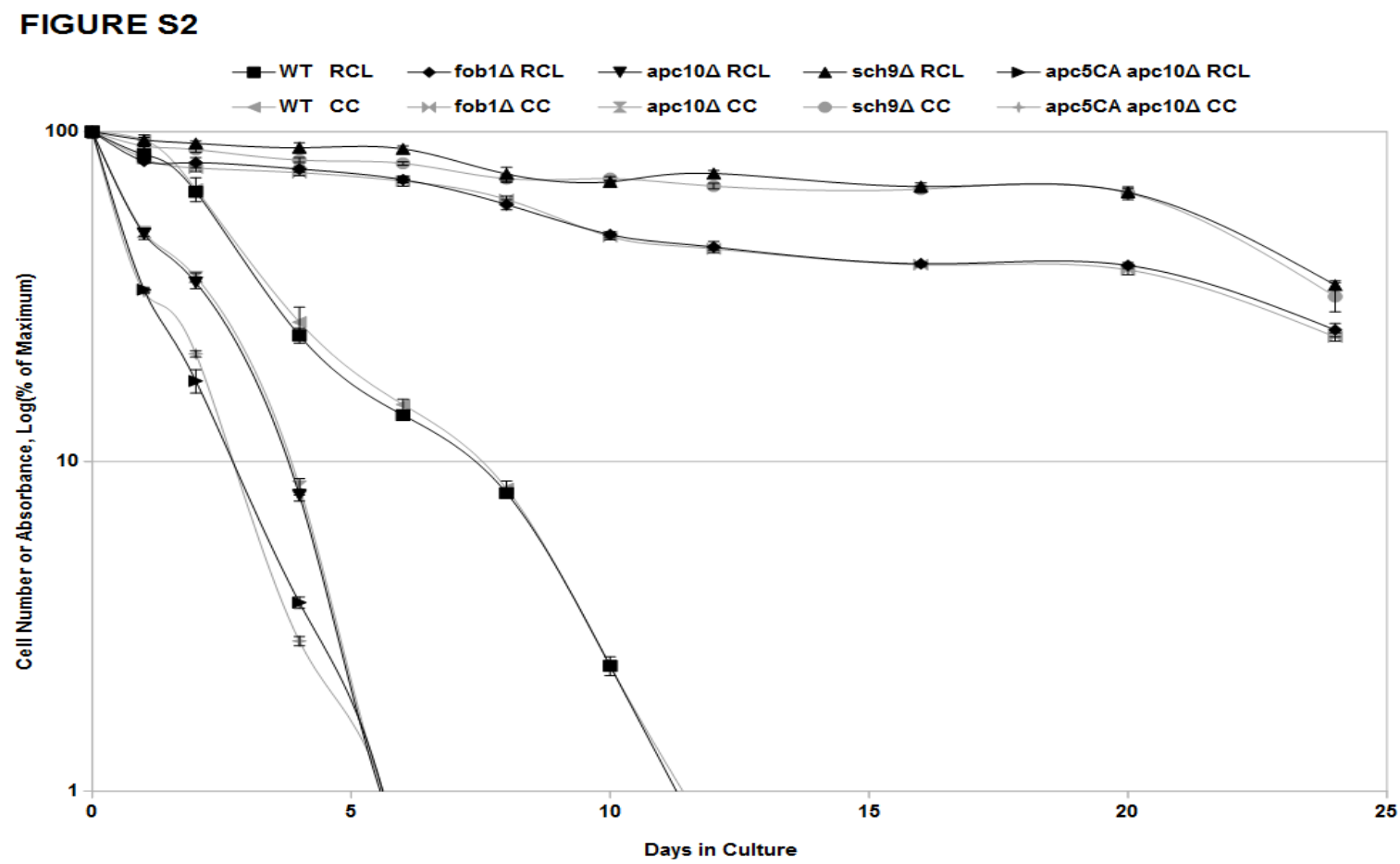

Figure S2. Analysis of long-lived and short-lived yeast mutants, log scale. Stationary phase aging cultures of various known long-lived or short-lived yeast strains were prepared in CM containing $2 \%$ glucose were incubated for the indicated number of days ( $x$-axis). Each day, and aliquot was removed from the culture and subjected to either the traditional CC assay (grey) or the novel RCL assay described here (black). For CC assays, viability is expressed as a percent of the mean cell number at day 0 , for $\mathrm{RCL}$ assays as logarithm of percent of the mean absorbance at day 0 ( $y$-axis). Strains are identified above the panel. Error bars represent the standard error of three independent replicates. 